\title{
Reversal of bronchiectasis caused by chronic aspiration in cri du chat syndrome
}

Bronchiectasis is a chronic lung disease whose pathophysiology is poorly understood. It has traditionally been considered "permanent and irreversible". In addition to chronic airway inflammation and infection, chronic aspiration has been implicated as another aetiological possibility. $^{2-4}$

We recently cared for a Samoan child with cri du chat syndrome, admitted four times with wheezing before 15

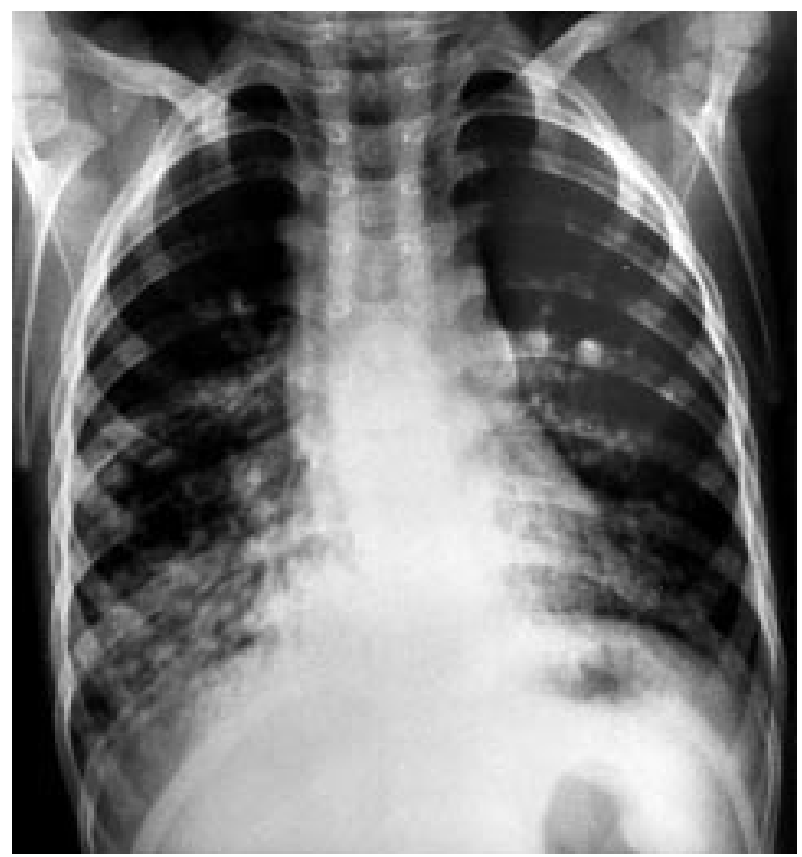

Figure 1 Frontal chest radiograph, age 2.5 years. There are bibasilar reticulonodular opacities. The heart borders are obscured, suggesting opacification in the right middle lobe and lingula.

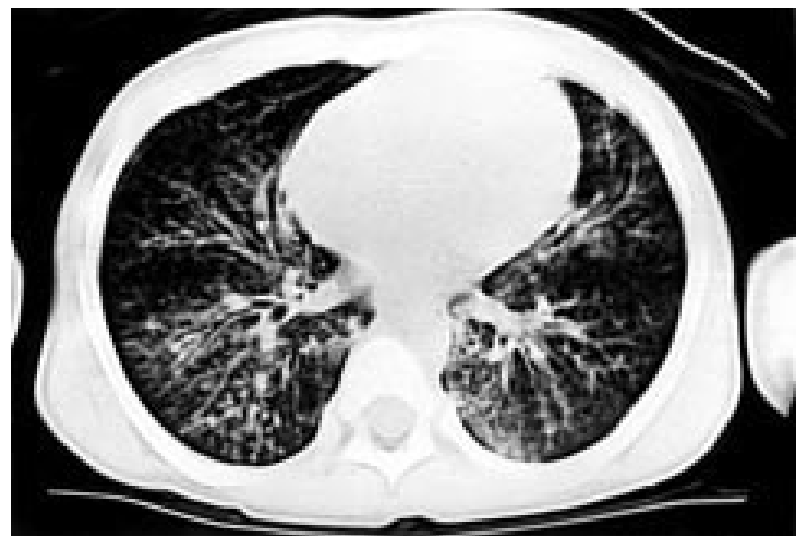

Figure 2 HRCT performed one week after fig 1. Axial thin section (1 mm) HRCT image through the lung bases shows dilated bronchi with thickened walls seen as "tram lines". This cylindrical type bronchiectasis was seen at multiple levels; images more superiorly confirmed the right middle lobe and lingular consolidations. months of age. He had persistent central and peripheral type wheezing and digital clubbing. A modified barium swallow revealed mild dysfunction of the oral phase of swallowing. A nuclear scintiscan revealed eight episodes of gastro-oesophageal reflux. Chest radiograph revealed bibasilar reticulonodular opacities with new right middle lobe disease (fig 1). High resolution computed tomography

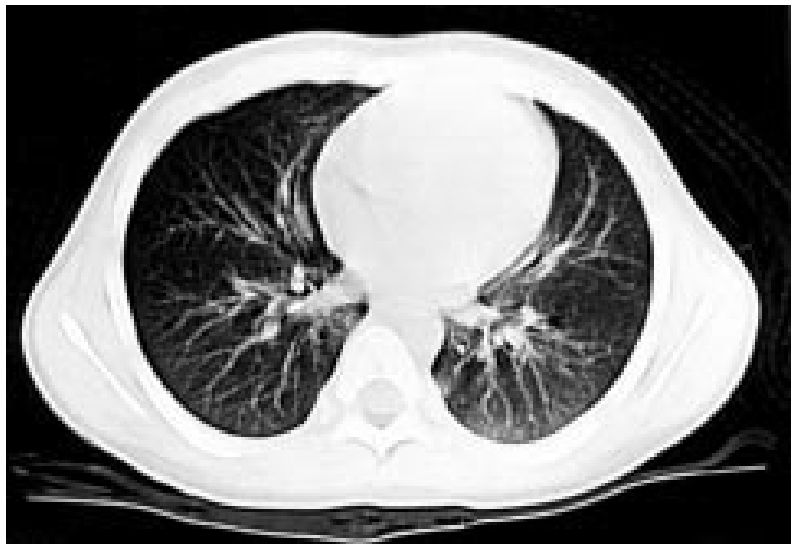

Figure 3 HRCT performed six months after surgery for gastro-oesophageal reflux (age 3 years). This section HRCT image at the same level as fig 2 shows notable interval improvement with minimal residual bronchiectatic changes in the right middle lobe.

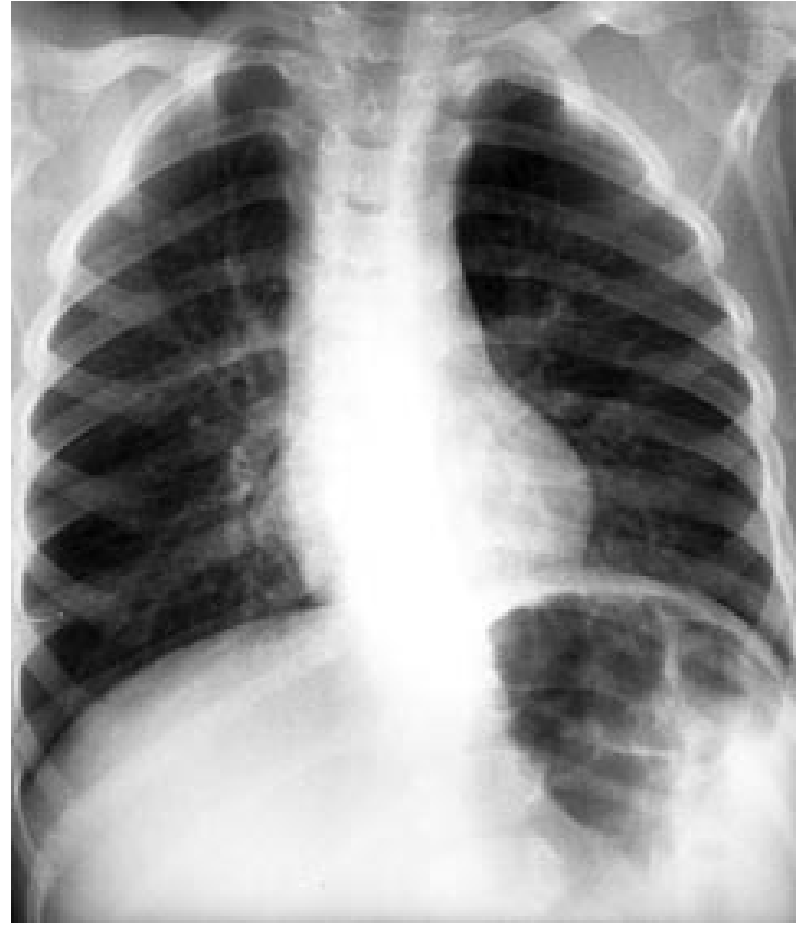

Figure 4 Frontal chest radiograph, age 3.5 years. There is no radiographic suggestion of bronchiectasis. 
(HRCT) scan of his chest showed cylindrical bronchiectasis (fig 2). Bronchoscopy with bronchoalveolar lavage revealed inflammatory cells, and staining of the lavage fluid with oil red-O revealed numerous lipid laden macrophages, suggestive of aspiration.

He was designated "nil by mouth" and underwent Nissen fundoplication with gastrostomy tube placement. Seven months post-procedure, a follow up HRCT scan of his chest showed near resolution of the bronchiectasis (fig 3). By age 3.5 years the digital clubbing had resolved; a chest radiograph showed no bronchiectasis (fig 4).

The term "bronchiectasis" has traditionally implied permanent, irreversible alteration in the anatomy of the airway. ${ }^{15}$ Chronic aspiration is a recognised condition that can lead to bronchiectasis in adults and children. ${ }^{56}$ Bronchiectasis has also been shown to be more common in patients of Polynesian descent, as a result of a suspected ciliary defect. ${ }^{7}$ The resolution of the symptoms and the radiographic evidence of bronchiectasis in this child underscore the importance of aggressive therapy for aspiration and concomitant bronchial hyperreactivity, and the prudence of watchful waiting in stable paediatric patients with bronchiectasis prior to surgical intervention, because of the possibility of recovery.
A C PITNEY

C W CALLAHAN

Department of Pediatrics, Tripler Army Medical Center,

Hawaii 96859-5000

L RUESS

Department of Radiology, Tripler Army Medical Center

Correspondence to: $\operatorname{Dr} C W$ Callahan, Pediatrics (MCHK-PE),

1 farrett White Road, Honolulu, HI 96859-5000

charles.callahan@amedd.army.mil

The views and opinions expressed in this manuscript are those of the authors and do not reflect the official policy or position of the Department of the Army, the Department of Defense, or the United States Government.

1 Reid L. Reduction in bronchial subdivision in bronchiectasis. Thorax 1950;5:233-47.

2 Brown MA, Lemen RJ. Bronchiectasis. In: Kendig's disorders of the respiratory tract in children, 6th edn. Philadelphia: WB Saunders, 1998:538-52.

3 Lewiston NJ. Bronchiectasis. In: Hilman BC, ed. Pediatric respiratory disease. Philadelphia: WB Saunders, 1993:222-9.

4 Hardy KA, Schidlow DV, Zaeri N. Obliterative bronchiolitis in children. Chest 1988;93:460-6.

5 Lewiston NJ. Bronchiectasis in childhood. Pediatr Clin North Am 1984;31:865-77.

6 Barker AF, Bardana EJ. Bronchiectasis: update of an orphan disease. Am Rev Respir Dis 1988;137:969-78.

7 Waite DA, Wakefield SJ, Moriarty KM, et al. Polynesian bronchiectasis. Eur f Respir Dis 1983;64S:31-6. 